\title{
On multiple Hurwitz zeta-function values at rational arguments
}

by

S. Kanemitsu (Fukuoka), Y. TAnigawa (Nagoya) and

M. Yoshimoto (Nagoya)

\section{Dedicated to Professor Iekata Shiokawa on his sixtieth birthday with great respect}

1. Introduction and corrected form of D. Klusch's formula. Entry 8, Chapter 15 of Ramanujan's celebrated second notebook [2] contains a "truly remarkable" ([3, p. 124]) formula which is "one of the most interesting and incredible formulas in the notebooks" ([2, pp. 50-51]):

ENTRY 8. For $\tau>0$,

$$
\text { (A) } \begin{aligned}
\sum_{n=1}^{\infty} \frac{1}{e^{\tau n^{2}}-1} & =\frac{\pi^{2}}{6 \tau}+\frac{1}{2} \sqrt{\frac{\pi}{\tau}} \zeta\left(\frac{1}{2}\right)+\frac{1}{4} \\
& +\sqrt{\frac{\pi}{2 \tau}} \sum_{n=1}^{\infty} \frac{1}{\sqrt{n}} \cdot \frac{\cos \left(2 \pi \sqrt{\frac{n \pi}{\tau}}+\frac{\pi}{4}\right)-e^{-2 \pi \sqrt{n \pi / \tau}} \cos \left(\frac{\pi}{4}\right)}{\cosh \left(2 \pi \sqrt{\frac{n \pi}{\tau}}\right)-\cos \left(2 \pi \sqrt{\frac{n \pi}{\tau}}\right)},
\end{aligned}
$$

where

$$
\zeta(s)=\sum_{n=1}^{\infty} \frac{1}{n^{s}}, \quad s=\sigma+i t, \sigma>1,
$$

denotes the Riemann zeta-function. We shall denote the complex variable $s=\sigma+i t$ throughout in what follows.

A character analogue of (A) was obtained by S. Egami [7] relating the value of $L(1 / 2, \chi)$, where $\chi$ is a primitive Dirichlet character $\bmod q$, to

$$
\sum_{r=1}^{q} \chi(r) \sum_{n=1}^{\infty} \frac{e^{-(r / q) n^{2} \tau}}{1-e^{-n^{2} \tau}} .
$$

Recently, M. Katsurada [14] has generalized all the preceding results to the framework of $r$-multiple Hurwitz zeta-function $\zeta_{r}(s, \alpha)$ (where $\alpha$ is a

2000 Mathematics Subject Classification: 11M35, 11M06. 
positive real parameter) defined by

$$
\zeta_{r}(s, \alpha)=\sum_{m_{1}, \ldots, m_{r}=0}^{\infty} \frac{1}{\left(m_{1}+\ldots+m_{r}+\alpha\right)^{s}}, \quad \sigma>r
$$

or equivalently by

$$
\zeta_{r}(s, \alpha)=\sum_{n=0}^{\infty} \frac{(r)_{n}}{n !}(n+\alpha)^{-s}, \quad \sigma>r
$$

where $(r)_{n}$ denotes the Pochhammer symbol or the rising factorial (cf. [6, p. 6])

$$
(x)_{n}=\frac{\Gamma(x+n)}{\Gamma(x)}=x(x+1) \ldots(x+n-1),
$$

where $\Gamma(s)$ denotes the gamma function.

The multiple Hurwitz zeta-function $\zeta_{r}(s, \alpha)$ is a special case of the Barnes multiple Hurwitz zeta-function $\zeta_{r}\left(s, \alpha,\left(\omega_{1}, \ldots, \omega_{r}\right)\right)$ with the base $\omega_{1}=$ $\ldots=\omega_{r}=1$ (cf. [1]). This special case, however, was introduced first by $\mathrm{Hj}$. Mellin [17] who already stated the important representation of $\zeta_{r}(s, \alpha)$ in terms of the ordinary Hurwitz zeta-function $\zeta_{1}(s, \alpha)=\zeta(s, \alpha)$,

$$
\zeta_{r}(s, \alpha)=\sum_{l=0}^{r-1} c_{r, l}(\alpha) \zeta(s-l, \alpha),
$$

where $c_{r, l}(\alpha)$ are Mellin-Barnes coefficients defined by (3.17) below and

$$
\zeta(s, \alpha)=\sum_{n=0}^{\infty}(n+\alpha)^{-s}, \quad \sigma>1, \quad \zeta(s, 1)=\zeta(s) .
$$

We note the slight inconsistency in the notation of the multiple Hurwitz zeta-function. In Mellin [17] our $\zeta_{r}(s, \alpha)$ is denoted by $\zeta_{r-1}(s, \alpha)$. Mellin did not give the coefficients explicitly. They were given in closed form by Choi [4], Vardi [19] and by the present authors [10].

Many authors including Choi, Quine, Srivastava, Vardi and Wilton have been concerned with the evaluation of power series with special values of $\zeta(s, \alpha)$ at positive integers $\geq 2$,

$$
\sum_{m=0}^{\infty} \frac{1}{m+\lambda} \zeta(m, \alpha) z^{m}
$$

but have been successful only for small values of $\lambda, \lambda=0,1,2$. Only our former paper [10] gives a complete answer to this problem.

Katsurada [14] applies his method of Mellin-Barnes integrals to generalize $(\mathrm{A})$ to $\zeta_{r}(1 / 2, \alpha)$. However, his result (Theorem 1 ) is explicit only for $r=1$ (because he did not evaluate the $r$ th derivative of the quantity explic- 
itly, which does not seem straightforward). In Section 5 we shall avoid this differentiation process by a new trick and give the explicit evaluation.

In Section 3, however, we shall make full use of the representation (1.5) to transfer our results in Section 2 (Theorem 2.1) on the closed form evaluation of the Hurwitz zeta-function at all negative rational points $b / a$ with $b$ odd and $a$ even, to that of the values of the multiple Hurwitz zeta-function at all positive rational points in $(0,1)$, so that our result (Theorem 3.1) gives not only an explicit formula for $\zeta_{r}(1 / 2, \alpha)$ but also a full result for $\zeta_{r}(b /(2 N), \alpha)$ with $b$ an odd integer.

We accomplish the evaluation of the Hurwitz zeta-function at negative rational arguments in a manner similar to that in [12], and so we may say our present investigation is in the same vein as before. (For possible choices of other gamma factors, cf. Koshlyakov [16].)

Our evaluations in Theorem 3.1, however, take a detour of moving to negative rational points and going back to positive rational points by decomposition (3.18) (in place of the customary functional equation), so that our result at first glance does not seem to imply Katsurada's result as it stands, since his result involves undetermined derivatives while in ours we determine the derivatives. In Section 4 we shall take a more direct approach to evaluating the multiple Hurwitz zeta-function at positive rational arguments.

In the following, interchanges of limit processes are justified by absolute convergence, and we shall not mention this at each occurrence.

In the remainder of this section, we shall present a corrected version of D. Klusch's result [15, formula (B)] and point out that the corrected formula is not very different from Euler's partial fraction expansion of the hyperbolic cotangent function (cf. [12]). The error occurs in the integrand defined by $[15,(2.7)]$, which should be $\psi(s)=\Gamma(s) \zeta(s) \zeta(-2 s)$, and so in [15, (2.10)], the integral $\frac{1}{2 \pi i} \int_{\left(c^{\prime}\right)} \psi(s) d s$ cannot be expanded in Lambert series (cf. [12] for more detailed exposition).

Proposition 1.1 (corrected version of D. Klusch's formula [15]). For $\tau>0$ we have

$$
\sum_{n=1}^{\infty}\left(\frac{\cosh \frac{\tau}{2 n^{2}}}{\sinh \frac{\tau}{2 n^{2}}}-\frac{2 n^{2}}{\tau}\right)=\frac{1}{2} \sqrt{\frac{\tau}{\pi}} \sum_{n=1}^{\infty} n^{-3 / 2} \frac{\sinh \sqrt{\frac{\pi \tau}{n}}-\sin \sqrt{\frac{\pi \tau}{n}}}{\cosh \sqrt{\frac{\pi \tau}{n}}-\cos \sqrt{\frac{\pi \tau}{n}}}
$$

Proof. First we introduce a general principle due to Liouville (cf. [16]).

PRINCIPLE. Suppose we have two absolutely convergent Dirichlet series

$$
\phi_{1}(s)=\sum_{n=1}^{\infty} \frac{a_{n}}{\lambda_{n}^{s}} \quad\left(\Re s>\sigma_{1}\right) \quad \text { and } \quad \phi_{2}(s)=\sum_{n=1}^{\infty} \frac{b_{n}}{\mu_{n}^{s}} \quad\left(\Re s>\sigma_{2}\right) \text {, }
$$

where $\sigma_{j}, j=1,2$, is the abscissa of absolute convergence of $\phi_{j}$. Then their 
product $\phi$ has the Dirichlet series expansion

$$
\phi(s)=\phi_{1}(s) \phi_{2}(s)=\sum_{n=1}^{\infty} \frac{c_{n}}{\nu_{n}^{s}}, \quad c_{n}=\sum_{\lambda_{l} \mu_{m}=\nu_{n}} a_{l} b_{m},
$$

in the same half-plane. Then an integral of the form $\left(\kappa>\sup \left(\sigma_{1}, \sigma_{2}\right)\right)$

$$
I(x)=\frac{1}{2 \pi i} \int_{(\kappa)} \Delta(s) \phi(s) x^{-s} d s,
$$

where $(\kappa)$ denotes the vertical line $\sigma=\kappa,-\infty<t<\infty(s=\sigma+i t)$, can be expanded in the form

$$
I(x)=\sum_{n=1}^{\infty} c_{n} \cdot \frac{1}{2 \pi i} \int_{(\kappa)} \Delta(s)\left(x \nu_{n}\right)^{-s} d s,
$$

so that we are led to the consideration of the integral

$$
\frac{1}{2 \pi i} \int_{(\kappa)} \Delta(s) X^{-s} d s
$$

which we designated as $E(X)$ in [11].

We apply this to the case of

$$
\zeta(s) \zeta(2 s-2)=\sum_{n=1}^{\infty} \frac{c_{n}}{n^{s}}, \quad c_{n}=\sum_{d \delta^{2}=n} \delta^{2},
$$

and evaluate the integral

$$
\begin{aligned}
I_{c}(\tau) & =\frac{\pi}{\tau} \cdot \frac{1}{2 \pi i} \int_{(c)} \frac{\left(\frac{2 \pi}{\tau}\right)^{-s}}{\sin \frac{\pi}{2} s} \zeta(s) \zeta(2 s-2) d s \\
& =\frac{\pi}{\tau} \sum_{n=1}^{\infty} c_{n} \cdot \frac{1}{2 \pi i} \int_{(c)} \frac{1}{\sin \frac{\pi}{2} s}(n X)^{-s} d s
\end{aligned}
$$

where $c>3 / 2$ and $X=2 \pi / \tau$.

Applying the known formula

$$
\frac{1}{2 \pi i} \int_{(c)} \frac{1}{\sin \frac{\pi z}{2}} \frac{d z}{\alpha^{z}}=\frac{2}{\pi} \cdot \frac{1}{1+\alpha^{2}}
$$

$(0<c<2, \alpha>0)$ (cf. [16, (3.3), p. 116]) to the integral in (1.8), we deduce that

$$
I_{c}(\tau)=\frac{2}{\tau} \sum_{n=1}^{\infty} \sum_{d \delta^{2}=n} \frac{\delta^{2}}{1+\left(d \delta^{2} \frac{2 \pi}{\tau}\right)^{2}},
$$

whence (by writing $m$ and $n$ for $d$ and $\delta$ respectively) 


$$
\begin{aligned}
I_{c}(\tau) & =\frac{2}{\tau} \sum_{n=1}^{\infty}\left(\frac{\tau}{2 \pi n}\right)^{2} \sum_{m=1}^{\infty} \frac{1}{m^{2}+\left(\frac{\tau}{2 \pi n^{2}}\right)^{2}} \\
& =\frac{2}{\tau} \sum_{m=1}^{\infty}\left(\frac{\tau}{2 \pi m}\right)^{2} \sum_{n=1}^{\infty} \frac{n^{2}}{\left(\frac{\tau}{2 \pi m}\right)^{2}+n^{4}} .
\end{aligned}
$$

Now, if we apply the well-known partial fraction formula of Euler

$$
\frac{1}{e^{2 \pi x}-1}=-\frac{1}{2}+\frac{1}{2 \pi x}+\frac{x}{\pi} \sum_{n=1}^{\infty} \frac{1}{x^{2}+n^{2}},
$$

in the form

$$
\sum_{n=1}^{\infty} \frac{1}{n^{2}+x^{2}}=\frac{\pi}{x}\left(\frac{1}{2} \operatorname{coth} \pi x-\frac{1}{2 \pi x}\right)
$$

to the first equality in (1.11), we get the left side of (1.7).

On the other hand, if we apply another identity

$$
\sum_{n=1}^{\infty} \frac{n^{2}}{a^{2}+n^{4}}=\frac{\pi}{2 \sqrt{2 a}} \cdot \frac{\sinh (\pi \sqrt{2 a})-\sin (\pi \sqrt{2 a})}{\cosh (\pi \sqrt{2 a})-\cos (\pi \sqrt{2 a})}
$$

$([15,(1.3)]$, cf. also [2]) to the second identity in (1.11), we obtain the right side of $(1.7)$.

REMARK 1. As is apparent from the above proof, formula (1.7) is just a computation of the double sum (1.10) as two different repeated simple sums by the use of Euler's partial fraction formula (1.12) and the identity (1.14) as the case may be. For a more instructive exposition, cf. [12].

\section{Values of Hurwitz zeta-function at negative rational $b / a$ with} $b$ odd, $a$ even. In this section, we shall use an argument similar to that used in proving Theorem 3 in [12] to prove the following result, which may be regarded as a quasi-modular relation.

TheOREM 2.1. Let $h \geq 0, l \geq 0$ and $N \geq 1$ be fixed integers with $h<N$, and let $\alpha$ be a positive parameter. Let

$$
\begin{aligned}
& a_{j}=\cos \left(\frac{\pi}{2 N}\left(\frac{1}{2}-j\right)\right), \quad b_{j}=\sin \left(\frac{\pi}{2 N}\left(\frac{1}{2}-j\right)\right), \\
& B_{j}(n, h, l)=-2 \pi \alpha n-\frac{\pi(2 h+1)}{2 N}\left(\frac{1}{2}-j\right)-\frac{\pi(l-1)}{2}, \\
& A(y)=\pi(2 \pi y)^{1 /(2 N)},
\end{aligned}
$$

and

$$
\begin{aligned}
& f_{j}(n, h, l, x) \\
& \quad=\frac{\cos \left(2 A(n / x) b_{j}+B_{j}(n, h, l)\right)-e^{-2 A(n / x) a_{j}} \cos \left(B_{j}(n, h, l)\right)}{\cosh \left(2 A(n / x) a_{j}\right)-\cos \left(2 A(n / x) b_{j}\right)} .
\end{aligned}
$$


Then for $x>0$ we have

$$
\sum_{n=1}^{\infty} \frac{1}{n^{2 N l-2 h}} \cdot \frac{\exp \left(-\alpha n^{2 N} x\right)}{1-\exp \left(-n^{2 N} x\right)}=P(x)+S(x, \alpha),
$$

where

$$
\begin{aligned}
P(x)= & \zeta(2 N(l+1)-2 h) x^{-1} \\
& +\sum_{j=0}^{l} \frac{(-1)^{j}}{j !} \zeta(-j, \alpha) \zeta(2 N(l-j)-2 h) x^{j} \\
& +\frac{1}{2 N} \Gamma\left(-l+\frac{2 h+1}{2 N}\right) \zeta\left(-l+\frac{2 h+1}{2 N}, \alpha\right) x^{l-\frac{2 h+1}{2 N}}
\end{aligned}
$$

and

$$
S(x, \alpha)=\frac{(-1)^{h}}{2 N}\left(\frac{2 \pi}{x}\right)^{-l+\frac{2 h+1}{2 N}} \sum_{j=-(N-1)}^{N-1} \sum_{n=1}^{\infty} f_{j}(n, h, l, x) n^{-1-l+\frac{2 h+1}{2 N}}
$$

where $\sum_{j}^{\prime \prime}$ means summation over $j=-(N-1),-(N-3), \ldots, N-3, N-1$.

Proof. Consider the integral, with $c>1$,

$$
I(x)=I_{c}(x)=\frac{1}{2 \pi i} \int \Gamma(s) \zeta(s, \alpha) \zeta(2 N(s+l)-2 h) x^{-s} d s .
$$

Applying the Principle of Section 1 with $\lambda_{n}=n+\alpha$ and $\mu_{n}=n^{2 N}$ to $\zeta(s, \alpha) \zeta(2 N(s+l)-2 h)$, we see that

$$
\begin{aligned}
I(x) & =\sum_{n=1}^{\infty} \frac{1}{n^{2 N l-2 h}} \sum_{m=0}^{\infty} \frac{1}{2 \pi i} \int_{(c)} \Gamma(s)\left((m+\alpha) n^{2 N}\right)^{-s} d s \\
& =\sum_{n=1}^{\infty} \frac{1}{n^{2 N l-2 h}} e^{-\alpha n^{2 N} x} \sum_{m=0}^{\infty} e^{-m n^{2 N} x}
\end{aligned}
$$

on using the well-known formula for the Mellin inversion

$$
\frac{1}{2 \pi i} \int_{(c)} \Gamma(s) X^{-s} d s=e^{-X} .
$$

Summing the geometric series in (2.7), we get the left-hand side of (2.3).

We now move the line of integration $(c)$ to $\left(-c^{\prime}\right)$, where $c^{\prime}>l$. The integrand has simple poles at

$s=1, \quad s=-m$ for $m=0,1, \ldots, l-1(s=-l$ also occurs for $h=0)$ and

$$
s=-l+\frac{2 h+1}{2 N} .
$$


The sum of the residues at these poles is given as $P(x)$ in (2.4). Note that although $j=l$ in the second sum does not appear for $h>0$, we may add that term with $j=l$ in this case as it is zero.

Hence

$$
I(x)=P(x)+J(x)
$$

where

$$
J(x)=I_{-c^{\prime}}(x)=\frac{1}{2 \pi i} \int_{\left(-c^{\prime}\right)} \Gamma(s) \zeta(s, \alpha) \zeta(2 N(s+l)-2 h) x^{-s} d s .
$$

We are going to evaluate $J(x)$ in the form of the series on the right-hand side of (2.3). To this end we eventually make the change of variables

$$
1-(2 N(s+l)-2 h)=s_{1},
$$

but we shall make it in two stages so as to recall the functional equation of the Hurwitz zeta-function.

Thus, putting $s=1-s_{1}$, we recall the functional equation (cf. e.g. [20] or [9])

$$
\zeta(1-s, \alpha)=\frac{\Gamma(s)}{(2 \pi)^{s}}\left\{e^{-\pi i s / 2} \psi(s, \alpha)+e^{\pi i s / 2} \psi(s,-\alpha)\right\},
$$

where

$$
\psi(s, \lambda)=\sum_{n=1}^{\infty} \frac{e^{2 \pi i \lambda n}}{n^{s}}
$$

denotes the Lerch zeta-function or the polylogarithm of complex exponential argument (as such, this is written $\ell_{s}(\lambda)$ ). Hence

$$
\Gamma(1-s) \zeta(1-s, \alpha)=\frac{\pi}{(2 \pi)^{s} \sin \pi s}\left\{e^{-\pi i s / 2} \psi(s, \alpha)+e^{\pi i s / 2} \psi(s,-\alpha)\right\} .
$$

On the other hand, using the functional equation in unsymmetric form

$$
\zeta(s)=2(2 \pi)^{s-1} \sin (\pi s / 2) \Gamma(1-s) \zeta(1-s),
$$

we deduce that

$$
\begin{aligned}
\zeta(2 N & (1-s+l)-2 h) \\
= & 2(2 \pi)^{2 N(1-s+l)-2 h-1}(-1)^{N(1+l)-h+1} \sin (\pi N s) \\
& \times \Gamma(1-2 N(1-s+l)+2 h) \zeta(1-2 N(1-s+l)+2 h) .
\end{aligned}
$$

Hence the integrand of

$$
J(x)=\frac{1}{2 \pi i} \int_{\left(1+c^{\prime}\right)} \Gamma(1-s) \zeta(1-s, \alpha) \zeta(2 N(1-s+l)-2 h) x^{-(1-s)} d s
$$

becomes

$$
\begin{aligned}
& (2 \pi)^{2 N(1-s+l)-2 h-s}(-1)^{N(1+l)-h+1} C_{N}(\pi s) \\
& \times\left\{e^{-\pi i s / 2} \psi(s, \alpha)+e^{\pi i s / 2} \psi(s,-\alpha)\right\} \Gamma(1-2 N(1+l)+2 h+2 N s) \\
& \times \zeta(1-2 N(1+l)+2 h+2 N s) x^{-1+s}
\end{aligned}
$$


where

$$
C_{N}(z)=\frac{\sin N z}{\sin z}=\frac{e^{i N z}-e^{-i N z}}{e^{i z}-e^{-i z}}=\sum_{j=-(N-1)}^{N-1} e^{i j z}
$$

Now making the change of variables

$$
1-2 N(1+l)+2 h+2 N s=s_{1}
$$

we obtain

$$
\begin{aligned}
& \text { (2.16) } \quad J(x)=(-1)^{h+l}\left(\frac{2 \pi}{x}\right)^{-l+\frac{2 h+1}{2 N}} \frac{1}{2 \pi i} \int_{\left(c_{1}\right)}\left(\frac{x}{(2 \pi)^{1+2 N}}\right)^{\frac{s_{1}}{2 N}} \\
& \times C_{N}\left(\frac{\pi\left(s_{1}-2 h-1\right)}{2 N}\right)\left\{e^{\frac{\pi i(2 h+1)}{4 N}-\frac{\pi i(l+1)}{2}} e^{-\frac{\pi i}{4 N} s_{1}} \psi\left(\frac{s_{1}-2 h-1}{2 N}+1+l, \alpha\right)\right. \\
& \left.+e^{-\frac{\pi i(2 h+1)}{4 N}+\frac{\pi i(l+1)}{2}} e^{\frac{\pi i}{4 N} s_{1}} \psi\left(\frac{s_{1}-2 h-1}{2 N}+1+l,-\alpha\right)\right\} \Gamma\left(s_{1}\right) \zeta\left(s_{1}\right) \frac{d s_{1}}{2 N}
\end{aligned}
$$

after simplification using

$$
C_{N}(z+\pi l)=(-1)^{(N-1) l} C_{N}(z) .
$$

We note that $c_{1}=1-2 N l+2 h+2 N c^{\prime}>1$ and hence that both zeta-functions can be expanded into Dirichlet series. We have therefore

$$
\begin{aligned}
& e^{-\frac{\pi i}{4 N} s_{1}} \psi\left(\frac{s_{1}-2 h-1}{2 N}+1+l, \alpha\right) \zeta\left(s_{1}\right) \\
& =\sum_{m, n=1}^{\infty} e^{2 \pi i \alpha n} n^{-1-l+\frac{2 h+1}{2 N}}\left(m n^{\frac{1}{2 N}} e^{\frac{\pi i}{4 N}}\right)^{-s_{1}}=G_{1}\left(s_{1}\right)
\end{aligned}
$$

say. Similarly

$$
\begin{aligned}
& e^{\frac{\pi i}{4 N} s_{1}} \psi\left(\frac{s_{1}-2 h-1}{2 N}+1+l,-\alpha\right) \zeta\left(s_{1}\right) \\
& =\sum_{m, n=1}^{\infty} e^{-2 \pi i \alpha n} n^{-1-l+\frac{2 h+1}{2 N}}\left(m n^{\frac{1}{2 N}} e^{-\frac{\pi i}{4 N}}\right)^{-s_{1}} \\
& =G_{2}\left(s_{1}\right)=\overline{G_{1}\left(\bar{s}_{1}\right)},
\end{aligned}
$$

say.

Hence, substituting (2.17) and (2.18) in (2.16) and writing $s$ for $s_{1}$ and $\kappa$ for $c_{1}$, we derive that

$$
J(x)=J_{1}(x)+J_{2}(x)
$$

where

$$
\begin{aligned}
(2.20) J_{1}(x)= & \frac{(-1)^{h+l}}{2 N}\left(\frac{2 \pi}{x}\right)^{-l+\frac{2 h+1}{2 N}} e^{\frac{\pi i(2 h+1)}{4 N}-\frac{\pi i(l+1)}{2}} \\
& \times \frac{1}{2 \pi i} \int_{(\kappa)}\left(\frac{x}{(2 \pi)^{1+2 N}}\right)^{\frac{s}{2 N}} C_{N}\left(\frac{\pi(s-2 h-1)}{2 N}\right) \Gamma(s) G_{1}(s) d s
\end{aligned}
$$


and

$$
\begin{aligned}
J_{2}(x) & =\frac{(-1)^{h+l}}{2 N}\left(\frac{2 \pi}{x}\right)^{-l+\frac{2 h+1}{2 N}} e^{-\frac{\pi i(2 h+1)}{4 N}+\frac{\pi i(l+1)}{2}} \\
& \times \frac{1}{2 \pi i} \int_{(\kappa)}\left(\frac{x}{(2 \pi)^{1+2 N}}\right)^{\frac{s}{2 N}} C_{N}\left(\frac{\pi(s-2 h-1)}{2 N}\right) \Gamma(s) G_{2}(s) d s .
\end{aligned}
$$

Changing the order of summation and integration, we obtain

$$
\begin{aligned}
J_{1}(x)= & \frac{(-1)^{h+l}}{2 N}\left(\frac{2 \pi}{x}\right)^{-l+\frac{2 h+1}{2 N}} e^{\frac{\pi i(2 h+1)}{4 N}-\frac{\pi i(l+1)}{2}} \\
& \times \sum_{m, n=1}^{\infty} e^{2 \pi i \alpha n} n^{-1-l+\frac{2 h+1}{2 N}} E\left(X_{m, n}\right),
\end{aligned}
$$

where

$$
E\left(X_{m, n}\right)=\frac{1}{2 \pi i} \int_{(\kappa)} X_{m, n}^{-s} C_{N}\left(\frac{\pi(s-2 h-1)}{2 N}\right) \Gamma(s) d s
$$

and

$$
X_{m, n}=2 \pi m\left(\frac{2 \pi n}{x}\right)^{\frac{1}{2 N}} e^{\frac{\pi i}{4 N}}
$$

and similarly

$$
\begin{aligned}
J_{2}(x)= & \frac{(-1)^{h+l}}{2 N}\left(\frac{2 \pi}{x}\right)^{-l+\frac{2 h+1}{2 N}} e^{-\frac{\pi i(2 h+1)}{4 N}+\frac{\pi i(l+1)}{2}} \\
& \times \sum_{m, n=1}^{\infty} e^{-2 \pi i \alpha n} n^{-1-l+\frac{2 h+1}{2 N}} E\left(\bar{X}_{m, n}\right) .
\end{aligned}
$$

Thus we are led to evaluate the integral $E\left(X_{m, n}\right)$. Recalling the definition (2.15) of $C_{N}(z)$, we see that

$$
X_{m, n}^{-s} C_{N}\left(\frac{\pi(s-2 h-1)}{2 N}\right)=\sum_{j=-(N-1)}^{N-1} e^{-\frac{\pi i(2 h+1)}{2 N} j}\left(X_{m, n} e^{-\frac{\pi i}{2 N} j}\right)^{-s}
$$

and that

$$
\left|\arg X_{m, n} e^{-\frac{\pi i}{2 N} j}\right|<\pi / 2
$$

Hence, by the fundamental Mellin inversion formula used above,

$$
\begin{aligned}
E\left(X_{m, n}\right) & =\sum_{j=-(N-1)}^{N-1} e^{-\frac{\pi i(2 h+1)}{2 N} j} e^{-X_{m, n} e^{-\frac{\pi i}{2 N} j}} \\
= & \sum_{j=-(N-1)}^{N-1} e^{-\frac{\pi i(2 h+1)}{2 N} j}\left(\exp \left(-2 \pi\left(\frac{2 \pi n}{x}\right)^{\frac{1}{2 N}} e^{\frac{\pi i}{2 N}\left(\frac{1}{2}-j\right)}\right)\right)^{m} .
\end{aligned}
$$


Substituting (2.25) in $(2.20)^{\prime}$ and summing over $m$, we derive that

$$
\begin{aligned}
J_{1}(x)= & \frac{(-1)^{h}}{2 N}\left(\frac{2 \pi}{x}\right)^{-l+\frac{2 h+1}{2 N}} e^{\frac{\pi i(2 h+1)}{4 N}+\frac{\pi i(l-1)}{2}} \sum_{j=-(N-1)}^{N-1} e^{-\frac{\pi i(2 h+1)}{2 N} j} \\
& \times \sum_{n=1}^{\infty} e^{2 \pi i \alpha n} n^{-1-l+\frac{2 h+1}{2 N}} \frac{\exp \left(-2 \pi\left(\frac{2 \pi n}{x}\right)^{\frac{1}{2 N}} \xi_{j}\right)}{1-\exp \left(-2 \pi\left(\frac{2 \pi n}{x}\right)^{\frac{1}{2 N}} \xi_{j}\right)}
\end{aligned}
$$

where we write

$$
\xi_{j}=e^{\frac{\pi i}{2 N}\left(\frac{1}{2}-j\right)}\left(=a_{j}+i b_{j}\right) .
$$

Using the notation (2.1), we further transform (2.26) into

$$
\begin{aligned}
J_{1}(x)= & \frac{(-1)^{h}}{2 N}\left(\frac{2 \pi}{x}\right)^{-l+\frac{2 h+1}{2 N}} \sum_{j=-(N-1)}^{N-1} \prime \prime \\
& \times \sum_{n=1}^{\infty} n^{-1-l+\frac{2 h+1}{2 N}} \frac{\exp \left(-i B_{j}(n, h, l)-A(n / x) \xi_{j}\right)}{2 \sinh \left(A(n / x) \xi_{j}\right)} .
\end{aligned}
$$

Similar reasoning yields

$$
\begin{aligned}
J_{2}(x)= & \frac{(-1)^{h}}{2 N}\left(\frac{2 \pi}{x}\right)^{-l+\frac{2 h+1}{2 N}} \sum_{j=-(N-1)}^{N-1} \prime \prime \\
& \times \sum_{n=1}^{\infty} n^{-1-l+\frac{2 h+1}{2 N}} \frac{\exp \left(i B_{j}(n, h, l)-A(n / x) \bar{\xi}_{j}\right)}{2 \sinh \left(A(n / x) \bar{\xi}_{j}\right)}\left(=\overline{J_{1}(x)}\right) .
\end{aligned}
$$

To conclude the assertion it suffices to calculate the sum of the exponential functions contained in (2.28) and (2.29), or

$$
\Re\left(\frac{\exp \left(-i B_{j}(n, h, l)-A(n / x)\left(a_{j}+i b_{j}\right)\right) \sinh \left(A(n / x)\left(a_{j}-i b_{j}\right)\right)}{\sinh \left(A(n / x)\left(a_{j}+i b_{j}\right)\right) \sinh \left(A(n / x)\left(a_{j}-i b_{j}\right)\right)}\right),
$$

which is seen to coincide with $f_{j}(n, h, l, x)$ given in $(2.2)$, and the proof is complete.

REMARK 2. We note that in the special case $N=1, h=0$, we have $j=0$ and $f_{0}(n, 0,0, x)$ coincides with Katsurada's $f_{0}(n / x, \alpha n)$, and therefore our Theorem reduces to Katsurada's Corollary 1.1 of [14]. We also point out a minor slip in Katsurada's proof of his Theorem 1. In expressing the quantities $X_{k, n}^{ \pm}$on p. 169, he drops the coefficients $i$ before $b_{k}(\alpha n)$. This derivation corresponds to those of (2.27) and (2.28).

\section{Values of the multiple Hurwitz zeta-function at rational ar-} guments $b /(2 N)$ with $b$ odd in the critical strip. In this section our main objective is to transform our Theorem 2.1 to the case of multiple Hurwitz zeta-functions. But before that we shall present a simple method for evaluating the derivatives in Katsurada's Theorem 1 of [14]. 
3.1. Performance of differentiation. First we introduce some notation. Let $[x]_{n}$ denote the falling factorial

$$
[x]_{n}=x(x-1) \ldots(x-n+1)=(-1)^{n}(-x)_{n},
$$

a companion to the rising factorial or Pochhammer symbol,

$$
(x)_{n}=\frac{\Gamma(x+n)}{\Gamma(x)}
$$

introduced by (1.4). Let $s(n, k)$ (resp. $S(n, k))$ denote the Stirling numbers of the first (resp. second) kind defined by

$$
\begin{aligned}
{[x]_{n} } & =\sum_{k=0}^{n} s(n, k) x^{k}, \\
x^{n} & =\sum_{k=0}^{n} S(n, k)[x]_{k}
\end{aligned}
$$

(cf. [6, p. 213, Theorem A and p. 204]).

We now turn to the first objective. Katsurada's method is outlined in Remark 5(iii) of our paper [12], and the main point is to evaluate $(\tau>0)$

$$
X_{k}=\frac{(2 \pi)^{1 / 2-k}}{2 \sqrt{\tau}}\left\{e^{-\frac{1}{2} \pi i(k+1 / 2)} Y_{k}+e^{\frac{1}{2} \pi i(k+1 / 2)} \bar{Y}_{k}\right\}
$$

in closed form where

$$
\begin{gathered}
Y_{k}=\sum_{n=1}^{\infty} \frac{e^{2 \pi i n \alpha}}{n^{k+1 / 2}} \sum_{m=1}^{\infty} F_{k}\left((2 \pi)^{3 / 2} e^{\pi i / 4} m n^{1 / 2} \tau^{-1 / 2}\right), \\
F_{k}(z)=\frac{2}{\sqrt{\pi}}\left(\frac{z}{2}\right)^{k+1 / 2} K_{k+1 / 2}(z),
\end{gathered}
$$

and $K_{k+1 / 2}(z)$ denotes the modified Bessel function of the first kind and of half-integral order. Then, instead of using the closed form expression for $K_{k+1 / 2}(z)([8])$, he had recourse to the initial form $(k=0,1, \ldots)$

$$
K_{k+1 / 2}(z)=(-1)^{k} \sqrt{\frac{\pi}{2 z}} z^{k+1}\left(\frac{d}{z d z}\right)^{k} \frac{e^{-z}}{z},
$$

thereby introducing uncalculated derivatives (Lemma 3).

If we differentiate in (3.7), we get the following closed form expression for $K_{k+1 / 2}(z)$ :

$$
K_{k+1 / 2}(z)=\sqrt{\frac{\pi}{2 z}} e^{-z} \sum_{j=0}^{k} \frac{(k+j) !}{(k-j) !}\left(\frac{1}{2 z}\right)^{j} .
$$

Using (3.8) in (3.6) leads to

$$
F_{k}(z)=2^{-k} \sum_{j=0}^{k} \frac{(2 k-j) !}{j !} 2^{j-k} z^{j} e^{-z}
$$


which transforms the inner sum (over $m$ ) in (3.5) into the form

$$
2^{-k} \sum_{j=0}^{k} \frac{(2 k-j) !}{j !} 2^{j-k}\left(\frac{2 \pi n}{\tau}\right)^{j / 2} e^{\pi i j / 4}(2 \pi)^{j} \sum_{m=1}^{\infty} m^{j} e^{-2 m A(n / \tau) \xi_{0}}
$$

where $A(n / \tau)=\pi(2 \pi n / \tau)^{1 / 2}$ and $\xi_{0}=e^{\pi i / 4}$ are special cases of $N=1$ of (2.1).

The inner sum can be computed (cf. $[12,(71)])$ in closed form as

$$
\begin{aligned}
\sum_{a=1}^{j+1}(a-1) ! S(j+ & 1, a) \frac{1}{\left(e^{2 A(n / \tau) \xi_{0}}-1\right)^{a}} \\
& =\sum_{a=1}^{j+1}(a-1) ! S(j+1, a) \frac{e^{-a A(n / \tau) \xi_{0}}}{\left(2 \sinh \left(A(n / \tau) \xi_{0}\right)\right)^{a}} .
\end{aligned}
$$

Since this closed form is obtained by taking the $j$ th derivative of a composite function in $e^{t}$ (cf. [18, p. 192]), we may say that our claim that the general case of Katsurada would follow from the differentiation of the case of the Hurwitz zeta-function is in a sense substantiated.

Now make the successive substitutions: (3.11) into (3.10), (3.10) into (3.5), and (3.5) into (3.4) to conclude that

$$
\begin{aligned}
X_{k}= & \frac{(2 \pi)^{1 / 2-k}}{\sqrt{\tau}} 2^{-k} \sum_{a=1}^{k+1}(a-1) ! \sum_{j=a-1}^{k} \frac{(2 k-j) !}{j !} 2^{j-k}\left(\frac{2 \pi}{\tau}\right)^{j / 2}(2 \pi)^{j} \\
& \times S(j+1, a) \sum_{n=1}^{\infty} \frac{1}{n^{k+1 / 2-j / 2}}\left(Z_{a, j, n}+\bar{Z}_{a, j, n}\right)
\end{aligned}
$$

where

$$
Z=Z_{a, j, n}=\frac{e^{-\frac{1}{4} \pi i(k+1 / 2)+\frac{\pi}{4} i j+2 \pi i n \alpha-a A(n / \tau) \xi_{0}}}{\left(2 \sinh \left(A(n / \tau) \xi_{0}\right)\right)^{a}} .
$$

Hence

$$
Z_{a, j, n}+\bar{Z}_{a, j, n}=B / A
$$

say, where

$$
A=\left(\cosh \left(2 A(n / \tau) a_{0}\right)-\cos \left(2 A(n / \tau) b_{0}\right)\right)^{a},
$$

with $a_{0}=\cos \pi / 4=1 / \sqrt{2}=b_{0}$ as special cases of $(2.1)$.

On the other hand, $B$ is the sum of

$$
2^{-a} e^{-\frac{1}{2} \pi i(k+1 / 2)+\frac{\pi}{4} i j+2 \pi i n \alpha}\left(e^{-A(n / \tau) \sqrt{2} i}-e^{-A(n / \tau) \sqrt{2}}\right)^{a}
$$

and its conjugate, or twice the real part of (3.16).

Expanding (3.16) and taking its real part, we complete the differentiation process in Katsurada's Theorem 1 of [14]. 
3.2. Transfer of the results. We restate (1.5) as a lemma.

Lemma 3.1 (Mellin-Vardi). Let

$$
c_{r, l}(\alpha)=\frac{(-1)^{r-1-l}}{(r-1) !} \sum_{j=l}^{r-1}\left(\begin{array}{l}
j \\
l
\end{array}\right) s(r, j+1) \alpha^{j-l} \quad \text { for } 0 \leq l \leq r-1 .
$$

Then

$$
\zeta_{r}(s, \alpha)=\sum_{l=0}^{r-1} c_{r, l}(\alpha) \zeta(s-l, \alpha)
$$

Proof. We rewrite the coefficients $(r)_{n} / n$ ! as

$$
\frac{1}{(r-1) !} \cdot \frac{1}{n}(n)_{r}
$$

and expand $(n)_{r}$ as

$$
(n)_{r}=\sum_{l=0}^{r-1}(-1)^{r+l+1} s(r, l+1) n^{l+1}
$$

(see $(3.1)$ and $(3.2))$. Then

$$
\zeta_{r}(s, \alpha)=\frac{1}{(r-1) !} \sum_{n=0}^{\infty}(n+\alpha)^{-s} \sum_{j=0}^{r-1}(-1)^{r+j+1} s(r, j+1) n^{j} .
$$

Substituting

$$
n^{j}=(n+\alpha-\alpha)^{j}=\sum_{l=0}^{j}\left(\begin{array}{l}
j \\
l
\end{array}\right)(n+\alpha)^{l}(-\alpha)^{j-l}
$$

into (3.19) and changing the order of summation we deduce (3.18), and the proof is complete.

We remark that J.-S. Choi [4] has made an extensive study of $\zeta_{r}(s, \alpha)$ using the decomposition (3.18).

We are now in a position to state the closed form expression for $\zeta_{r}\left(\frac{2 h+1}{2 N}, \alpha\right), 0 \leq h \leq N-1$.

THEOREM 3.1. With the same notation as in Theorem 2.1, we have, for $x>0$,

$$
\begin{aligned}
\Gamma\left(\frac{2 h+1}{2 N}\right) \zeta_{r} & \left.\frac{2 h+1}{2 N}, \alpha\right)=\sum_{l=0}^{r-1}(-1)^{l}\left(1-\frac{2 h+1}{2 N}\right) c_{r, l}(\alpha) \\
& \times\left\{(-1)^{h+1}(2 \pi)^{-l+\frac{2 h+1}{2 N}} \sum_{j=-(N-1)}^{N-1} \sum_{n=1}^{\infty} f_{j}(n, h, l, x) n^{-1-l+\frac{2 h+1}{2 N}}\right.
\end{aligned}
$$




$$
\begin{aligned}
& +2 N x^{-l+\frac{2 h+1}{2 N}}\left(\sum_{n=1}^{\infty} \frac{1}{n^{2 N l-2 h}} \cdot \frac{\exp \left(-\alpha n^{2 N} x\right)}{1-\exp \left(-n^{2 N} x\right)}-\zeta(2 N(l+1)-2 h) x^{-1}\right. \\
& \left.\left.-\sum_{j=0}^{l} \frac{(-1)^{j}}{j !} \zeta(-j, \alpha) \zeta(2 N(l-j)-2 h) x^{j}\right)\right\} .
\end{aligned}
$$

The proof follows on substituting the results of Theorem 2.1 in

$$
\zeta_{r}\left(\frac{2 h+1}{2 N}, \alpha\right)=\sum_{l=0}^{r-1} c_{r, l}(\alpha) \zeta\left(-l+\frac{2 h+1}{2 N}, \alpha\right) .
$$

REMARK 3. (i) Katsurada [14] also proves a similar result for the Hurwitz-Lerch zeta-function

$$
\phi(s, \lambda, \alpha)=\sum_{n=0}^{\infty} \frac{e^{2 \pi i n \lambda}}{(n+\alpha)^{s}}
$$

corresponding to our Theorem 2.1. We may extend our results to this case.

The $\chi$-analogue of the above results must be more interesting, and we shall establish a $\chi$-analogue of Theorem 3.1 in our forthcoming paper [13] (cf. Katsurada's Corollary 2.2).

(ii) It should be pointed out that Katsurada's Theorem 3 of [14] giving the residue of the multiple Hurwitz zeta-function was already given by Barnes [1] and Choi [5].

4. Values of the $r$-multiple Hurwitz zeta-function at positive rational arguments $b / a$ with $b$ odd and $a$ even (revisited). As we stated in Introduction, our formula for $\zeta_{r}(b /(2 N), \alpha)$ in Theorem 3.1 take a detour of moving to negative rational points and going back to positive rational points by (1.5). In this section, we shall give a more direct approach to evaluating the multiple Hurwitz zeta-function at positive rational arguments, therewith revealing why Katsurada's method was successful only for $1 / 2$ (see Remark 4(i)). Our result does not involve derivatives.

The lines of argument will remain the same as in former papers [12] and [13] in that we are in pursuit of a relation similar to the modular relation: we incorporate the simple gamma factor $\Gamma(s)$, which is not the proper gamma factor appearing in the functional equation for the (product of) zeta-function under consideration.

Our formulation will be similar to that of Katsurada, but we will follow closely the proof of Theorem 2.1 in Section 2. In particular, we shall indicate those formulas which correspond to former ones in Section 2 by attaching an asterisk to formula numbers.

To formulate our result, we shall need to introduce new notation. First we define the new coefficients $Q_{l, k}(a, b)$ by 


$$
Q_{l, k}(a, b)=k ! \sum_{j=l}^{k}(-1)^{k-j} a^{j} S(j, l)\left\{c_{k+1, j}(b)-c_{k, j}(b)\right\},
$$

where $S(n, k)$ and $c_{r, l}(\alpha)$ are Stirling numbers and Mellin-Vardi coefficients defined by (3.3) and (3.17) respectively.

We also introduce Nörlund's generalized Bernoulli polynomials $B_{k}^{(r)}(\alpha)$, as in [14], by

$$
\left(\frac{z}{e^{z}-1}\right)^{r} e^{\alpha z}=\sum_{k=0}^{\infty} \frac{B_{k}^{(r)}(\alpha)}{k !} z^{k}, \quad|z|<2 \pi .
$$

The Mellin-Vardi coefficients and the Nörlund generalized Bernoulli polynomials are linked by

$$
B_{r-1-k}^{(r)}(\alpha)=k !(r-1-k) ! c_{r, k}(\alpha)=\left(\begin{array}{c}
r-1 \\
k
\end{array}\right)^{-1} \sum_{j=k}^{r-1}\left(\begin{array}{l}
j \\
k
\end{array}\right) s(r, j+1) \alpha^{j-k}
$$

as is seen by comparing

$$
\underset{s=k+1}{\operatorname{Res}} \zeta_{r}(s, \alpha)=c_{r, k}(\alpha), \quad k=0, \ldots, r-1,
$$

a consequence of (3.18) on the one hand, and

$$
\operatorname{Res}_{s=k+1} \zeta_{r}(s, \alpha)=\frac{(-1)^{r-k-1} B_{r-1-k}^{(r)}(\alpha)}{k !(r-1-k) !},
$$

on the other, which is due to E. W. Barnes [1], J.-S. Choi [4] and [14, Theorem 3].

Theorem 4.1. Let $h \geq 0$ and $N \geq 1$ be fixed integers $h<N$, and let $\alpha$ be a positive parameter. Further let

$$
\begin{aligned}
& a_{j}=\cos \left(\frac{\pi}{2 N}\left(\frac{1}{2}-j\right)\right), \quad b_{j}=\sin \left(\frac{\pi}{2 N}\left(\frac{1}{2}-j\right)\right) \\
& B^{*}(n, j, l, k)=-2 \pi \alpha n-\frac{\pi(2 h+1+l)}{2 N}\left(\frac{1}{2}-j\right)+\frac{\pi(k+1)}{2} \\
& A(y)=\pi(2 \pi y)^{\frac{1}{2 N}}
\end{aligned}
$$

and

$$
\begin{aligned}
& f^{*}(n, a, j, l, k, x) \\
= & 2^{1-a_{\Re}}\left\{e^{-i\left(B^{*}(n, j, l, k)\right)}\left(\frac{e^{-2 i A(n / x) b_{j}}-e^{-2 A(n / x) a_{j}}}{\cosh \left(2 A(n / x) a_{j}\right)-\cos \left(2 A(n / x) b_{j}\right)}\right)^{a}\right\} .
\end{aligned}
$$

Then for $x>0$ we have

$$
\sum_{n=1}^{\infty} n^{2 h} \frac{e^{-\alpha n^{2 N} x}}{\left(1-e^{-n^{2 N} x}\right)^{r}}=P(x)+S_{r}(x, \alpha)
$$


where

$$
\begin{aligned}
P(x)= & \sum_{j=0}^{r} \frac{(-1)^{r-j} B_{r-j}^{(r)}(\alpha)}{(r-j) !} \zeta(2 N j-2 h) x^{-j} \\
& +\frac{1}{2 N} \Gamma\left(\frac{2 h+1}{2 N}\right) \zeta_{r}\left(\frac{2 h+1}{2 N}, \alpha\right) x^{-\frac{2 h+1}{2 N}}
\end{aligned}
$$

and

$$
\begin{aligned}
S_{r}(x, \alpha)= & \frac{(-1)^{h}}{2 N}\left(\frac{2 \pi}{x}\right)^{\frac{2 h+1}{2 N}} \sum_{k=0}^{r-1} \frac{(-1)^{r-k-1} B_{r-k-1}^{(r)}(\alpha)}{k !(r-k-1) !(2 \pi)^{k}} \\
& \times \sum_{l=0}^{k}(-1)^{k-l} Q_{l, k}\left(\frac{1}{2 N}, \frac{2 N-2 h-1}{2 N}\right)(2 \pi)^{l}\left(\frac{2 \pi}{x}\right)^{\frac{l}{2 N}} \\
& \times \sum_{j=-(N-1)}^{N-1} \sum_{a=1}^{l+1}(a-1) ! S(l+1, a) \\
& \times \sum_{n=1}^{\infty} f^{*}(n, a, j, l, k, x) n^{\frac{2 h+1+l}{2 N}-k-1}
\end{aligned}
$$

where $\sum_{j}^{\prime \prime}$ denotes summation over $j=-(N-1),-(N-3), \ldots, N-3, N-1$.

Proof. Consider the integral, with $c_{0}>r$,

$$
I(x)=\frac{1}{2 \pi i} \int_{\left(c_{0}\right)} \Gamma(s) \zeta_{r}(s, \alpha) \zeta(2 N s-2 h) x^{-s} d s,
$$

where $\left(c_{0}\right)$ denotes the vertical line $s=\sigma+i t, \sigma=c_{0},-\infty<t<\infty$.

Applying the Principle of Section 1 to $\zeta_{r}(s, \alpha) \zeta(2 N s-2 h)$ and arguing in a manner similar to that in $[12, \S 3]$, we obtain

$$
I(x)=\sum_{n=1}^{\infty} n^{2 h} \frac{e^{-\alpha n^{2 N} x}}{\left(1-e^{-n^{2 N} x}\right)^{r}},
$$

i.e. the left-hand side of $(4.3)^{*}$.

We now shift the line of integration to $(-c)$ with $c>0$. In so doing, we encounter the simple poles of the integrand at $s=0, s=1, \ldots, r$ and $s=(2 h+1) /(2 N)$, which are the poles of $\Gamma, \zeta_{r}$ and $\zeta$, respectively (note that other poles of $\Gamma$ at negative integer arguments are cancelled by the zeros of $\zeta$ ).

Hence

$$
I(x)=P(x)+J(x),
$$

where

$$
J(x)=\frac{1}{2 \pi i} \int_{(-c)} \Gamma(s) \zeta_{r}(s, \alpha) \zeta(2 N s-2 h) x^{-s} d s
$$


and

$$
\begin{aligned}
P(x)= & \zeta_{r}(0, \alpha) \zeta(-2 h)+\sum_{j=1}^{r} \frac{(-1)^{r-j} B_{r-j}^{(r)}(\alpha)}{(r-j) !} \zeta(2 N j-2 h) x^{-j} \\
& +\frac{1}{2 N} \Gamma\left(\frac{2 h+1}{2 N}\right) \zeta_{r}\left(\frac{2 h+1}{2 N}, \alpha\right) x^{-\frac{2 h+1}{2 N}}
\end{aligned}
$$

which is seen to coincide with $(4.4)^{*}$ in view of the known formula

(cf. e.g. [14]).

$$
\zeta_{r}(0, \alpha)=\frac{(-1)^{r}}{r !} B_{r}^{(r)}(\alpha)
$$

The remaining task is to evaluate the integral $J(x)$ in the form as given by $(4.5)^{*}$. As in Section 2, we make the change of variables $s \leftrightarrow 1-s$ and use the functional equation for $\zeta_{r}(s, \alpha)$ in the form by Katsurada [14]

$$
\begin{aligned}
\zeta_{r}(1-s, \alpha)= & \sum_{k=0}^{r-1} \frac{(-1)^{r-k-1} B_{r-k-1}^{(r)}(\alpha)}{k !(r-k-1) !} \cdot \frac{\Gamma(s+k)}{(2 \pi)^{s+k}} \\
& \times\left\{e^{-\pi i(s+k) / 2} \psi(s+k, \alpha)+e^{\pi i(s+k) / 2} \psi(s+k,-\alpha)\right\},
\end{aligned}
$$

where

$$
\psi(s, \alpha)=\sum_{n=1}^{\infty} \frac{e^{2 \pi i n \alpha}}{n^{s}}
$$

denotes the Lerch zeta-function or the polylogarithm of complex exponential argument. Hence

$$
\begin{aligned}
& \Gamma(1-s) \zeta_{r}(1-s, \alpha)=\sum_{k=0}^{r-1} \frac{(-1)^{r-k-1} B_{r-k-1}^{(r)}(\alpha)}{k !(r-k-1) !} \cdot \frac{(s)_{k}}{(2 \pi)^{s+k}} \cdot \frac{\pi}{\sin \pi s} \\
& \times\left\{e^{-\pi i(s+k) / 2} \psi(s+k, \alpha)+e^{\pi i(s+k) / 2} \psi(s+k,-\alpha)\right\} .
\end{aligned}
$$

On the other hand, the functional equation for the Riemann zeta-function yields

$$
\begin{aligned}
\zeta(2 N(1-s)-2 h) & =(-1)^{N-h+1} 2(2 \pi)^{2 N(1-s)-2 h-1} \sin (\pi N s) \\
\times & \Gamma(1-2 N(1-s)+2 h) \zeta(1-2 N(1-s)+2 h) .
\end{aligned}
$$

Hence the integrand of

$$
J(x)=\frac{1}{2 \pi i} \int_{(1+c)} \Gamma(1-s) \zeta_{r}(1-s, \alpha) \zeta(2 N(1-s)-2 h) x^{s-1} d s
$$

becomes

$$
\sum_{k=0}^{r-1} \frac{(-1)^{r-k-1} B_{r-k-1}^{(r)}(\alpha)}{k !(r-k-1) !} G_{k}(s) x^{s-1}
$$

say, where 
$(4.14)_{2}^{*}$

$$
\begin{aligned}
G_{k}(s)= & (-1)^{N-h+1}(2 \pi)^{2 N(1-s)-2 h-s-k}(s)_{k} C_{N}(\pi s) \\
& \times\left\{e^{-\pi i(s+k) / 2} \psi(s+k, \alpha)+e^{\pi i(s+k) / 2} \psi(s+k,-\alpha)\right\} \\
& \times \Gamma(1-2 N(1-s)+2 h) \zeta(1-2 N(1-s)+2 h),
\end{aligned}
$$

and where

$$
C_{N}(z)=\frac{\sin N z}{\sin z}=\sum_{j=-(N-1)}^{N-1} e^{i j z} .
$$

We note that the line of integration being $(1+c)$, we have both $1+$ $2 N c+2 h>1$ and $c+1+k>1(0 \leq k \leq r-1)$, so that both $\zeta$ and $\psi$ are expansible in Dirichlet series.

Now make the change of variables

$$
1-2 N(1-s)+2 h=s_{1}, \quad s=\frac{s_{1}}{2 N}+1-\frac{2 h+1}{2 N} .
$$

Then $G_{k}(s) x^{s-1}$ becomes

$$
\begin{aligned}
G_{k}( & \left.\frac{s_{1}}{2 N}+1-\frac{2 h+1}{2 N}\right) x^{\frac{s_{1}}{2 N}-\frac{2 h+1}{2 N}} \\
= & (-1)^{h}(2 \pi)^{-k}\left(\frac{2 \pi}{x}\right)^{\frac{2 h+1}{2 N}}\left(\frac{x}{(2 \pi)^{2 N+1}}\right)^{\frac{s_{1}}{2 N}} \\
& \times\left(\frac{s_{1}}{2 N}+1-\frac{2 h+1}{2 N}\right)_{k} C_{N}\left(\frac{\pi\left(s_{1}-2 h-1\right)}{2 N}\right) \Gamma\left(s_{1}\right) \zeta\left(s_{1}\right) \\
& \times\left\{e^{-\frac{\pi i\left(s_{1}-2 h-1\right)}{4 N}-\frac{\pi i(k+1)}{2}} \psi\left(\frac{s_{1}-2 h-1}{2 N}+k+1, \alpha\right)\right. \\
& \left.+e^{\frac{\pi i\left(s_{1}-2 h-1\right)}{4 N}+\frac{\pi i(k+1)}{2}} \psi\left(\frac{s_{1}-2 h-1}{2 N}+k+1,-\alpha\right)\right\} .
\end{aligned}
$$

Thus, in order to obtain a closed form of $J(x)$, we will eventually need the evaluation of the integral of

$$
\frac{1}{2 \pi i} \int_{(\kappa)} X^{-s} C_{N}\left(\frac{\pi(s-2 h-1)}{2 N}\right)\left(\frac{s}{2 N}+\frac{a}{2 N}\right)_{k} \Gamma(s) d s .
$$

We shall use the following lemma (proof being given at the end) to transform the rising factorial in a tractable form.

Lemma 4.1. For any $a(\neq 0), b$ and $x$ we have

$$
(a x+b)_{k}=\sum_{l=0}^{k}(-1)^{k-l} Q_{l, k}(a,-b)(x)_{l},
$$

where $Q_{l, k}(a, b)$ is given at the beginning of this section. 
Now incorporating all the preceding, we successively get

$$
\begin{aligned}
(4.19)^{*} J(x)= & \sum_{k=0}^{r-1} \frac{(-1)^{r-k-1} B_{r-k-1}^{(r)}(\alpha)}{k !(r-k-1) !} \cdot \frac{1}{2 \pi i} \int_{(1+c)} G_{k}(s) x^{s-1} d s \\
= & (-1)^{h}\left(\frac{2 \pi}{x}\right)^{\frac{2 h+1}{2 N}} \sum_{k=0}^{r-1} \frac{(-1)^{r-k-1} B_{r-k-1}^{(r)}(\alpha)}{k !(r-k-1) !}(2 \pi)^{-k} \\
& \times \sum_{l=0}^{k}(-1)^{k-l} Q_{l, k}\left(\frac{1}{2 N}, \frac{2 N-2 h-1}{2 N}\right) \\
& \times \frac{1}{2 \pi i} \int_{\left(c_{1}\right)}\left(\frac{x}{(2 \pi)^{2 N+1}}\right)^{\frac{s}{2 N}} C_{N}\left(\frac{\pi(s-2 h-1)}{2 N}\right)(s)_{l} \Gamma(s) \\
& \times \zeta(s)\left\{e^{-\frac{\pi i(s-2 h-1)}{4 N}-\frac{\pi i(k+1)}{2}} \psi\left(\frac{s-2 h-1}{2 N}+k+1, \alpha\right)\right. \\
& \left.+e^{\frac{\pi i(s-2 h-1)}{4 N}+\frac{\pi i(k+1)}{2}} \psi\left(\frac{s-2 h-1}{2 N}+k+1,-\alpha\right)\right\} \frac{d s}{2 N} \\
= & \frac{(-1)^{h}}{2 N}\left(\frac{2 \pi}{x}\right)^{\frac{2 h+1}{2 N}} \sum_{k=0}^{r-1} \frac{(-1)^{r-k-1} B_{r-k-1}^{(r)}(\alpha)}{k !(r-k-1) !}(2 \pi)^{-k} \\
& \times \sum_{l=0}^{k}(-1)^{k-l} Q_{l, k}\left(\frac{1}{2 N}, \frac{2 N-2 h-1}{2 N}\right)\left\{J_{1}(x)+J_{2}(x)\right\}
\end{aligned}
$$

where

$$
\begin{array}{ll}
(4.20)^{*} & J_{1}(x)=e^{\frac{\pi i(2 h+1)}{4 N}-\frac{\pi i(k+1)}{2}} \sum_{n, m=1}^{\infty} e^{2 \pi i n \alpha} n^{\frac{2 h+1}{2 N}-k-1} E\left(X_{m, n}\right), \\
(4.21)^{*} & J_{2}(x)=e^{-\frac{\pi i(2 h+1)}{4 N}+\frac{\pi i(k+1)}{2}} \sum_{n, m=1}^{\infty} e^{-2 \pi i n \alpha} n^{\frac{2 h+1}{2 N}-k-1} E\left(\bar{X}_{m, n}\right)
\end{array}
$$

and (with $\kappa=c_{1}=1+2 N c+2 h$ )

$$
E\left(X_{m, n}\right)=\frac{1}{2 \pi i} \int_{(\kappa)} X_{m, n}^{-s} C_{N}\left(\frac{\pi(s-2 h-1)}{2 N}\right) \Gamma(s+l) d s
$$

with $X_{m, n}$ denoting

$$
X_{m, n}=2 \pi m\left(\frac{2 \pi n}{x}\right)^{\frac{1}{2 N}} e^{\frac{\pi i}{4 N}} .
$$

To evaluate $E\left(X_{m, n}\right)$ we use $(4.15)^{*}$ to write

$(4.24)^{*} \quad X_{m, n}^{-s} C_{N}\left(\frac{\pi(s-2 h-1)}{2 N}\right)=\sum_{j=-(N-1)}^{N-1} e^{-\frac{\pi i(2 h+1)}{2 N} j}\left(X_{m, n} e^{-\frac{\pi i}{2 N} j}\right)^{-s}$ 
with

$$
\left|\arg X_{m, n} e^{-\frac{\pi i}{2 N} j}\right|<\pi / 2
$$

Then the Mellin inversion formula

$$
\frac{1}{2 \pi i} \int_{(\kappa)} \Gamma(s+l) Y^{-s} d s=Y^{l} e^{-Y}
$$

yields

$$
\begin{aligned}
E\left(X_{m, n}\right)= & \left(2 \pi\left(\frac{2 \pi n}{x}\right)^{\frac{1}{2 N}} e^{\frac{\pi i}{4 N}}\right)^{l} \sum_{j=-(N-1)}^{N-1} e^{-\frac{\pi i(2 h+1+l)}{2 N} j} \\
& \times m^{l} \exp \left(-2 \pi m\left(\frac{2 \pi n}{x}\right)^{\frac{1}{2 N}} e^{\frac{\pi i}{2 N}(1 / 2-j)}\right) .
\end{aligned}
$$

Substituting $(4.25)^{*}$ in $(4.20)^{*}$ and summing over $m$ by appealing to the formula

$$
\sum_{m=1}^{\infty} m^{a-1} e^{-m \xi}=\sum_{l=1}^{a}(l-1) ! S(a, l) \frac{1}{\left(e^{\xi}-1\right)^{l}}
$$

(cf. e.g. $[11,(57)])$, we obtain

where we write

$$
\begin{aligned}
(4.26)^{*} \quad J_{1}(x)= & (2 \pi)^{l}\left(\frac{2 \pi}{x}\right)^{\frac{l}{2 N}} \sum_{j=-(N-1)}^{N-1} e^{\frac{\pi i(2 h+1+l)}{2 N}(1 / 2-j)-\frac{\pi i(k+1)}{2}} \\
& \times \sum_{n=1}^{\infty} e^{2 \pi i n \alpha} n^{\frac{2 h+1+l}{2 N}-k-1} \\
& \times \sum_{a=1}^{l+1}(a-1) ! \frac{S(l+1, a)}{\left(\exp \left(2 \pi\left(\frac{2 \pi n}{x}\right)^{\frac{1}{2 N}} \xi_{j}\right)-1\right)^{a}}
\end{aligned}
$$
$(4.27)^{*}$

$$
\xi_{j}=e^{\frac{\pi i}{2 N}(1 / 2-j)}\left(=a_{j}+i b_{j}\right) .
$$

Using the notation $(4.1)^{*}$, we may further transform $(4.26)^{*}$ into the form

$$
\begin{aligned}
(4.28)^{*} \quad J_{1}(x)= & (2 \pi)^{l}\left(\frac{2 \pi}{x}\right)^{\frac{l}{2 N}} \sum_{j=-(N-1)}^{N-1} \sum_{a=1}^{l+1}(a-1) ! S(l+1, a) \\
& \times \sum_{n=1}^{\infty} n^{\frac{2 h+1+l}{2 N}-k-1} \frac{\exp \left(-i B^{*}(n, j, l, k)-a A(n / x) \xi_{j}\right)}{\left(2 \sinh \left(A(n / x) \xi_{j}\right)\right)^{a}} .
\end{aligned}
$$

Similarly, we deduce that

$$
\begin{aligned}
(4.29)^{*} \quad J_{2}(x)= & (2 \pi)^{l}\left(\frac{2 \pi}{x}\right)^{\frac{l}{2 N}} \sum_{j=-(N-1)}^{N-1} \sum_{a=1}^{l+1}(a-1) ! S(l+1, a) \\
& \times \sum_{n=1}^{\infty} n^{\frac{2 h+1+l}{2 N}-k-1} \frac{\exp \left(i B^{*}(n, j, l, k)-a A(n / x) \bar{\xi}_{j}\right)}{\left(2 \sinh \left(A(n / x) \bar{\xi}_{j}\right)\right)^{a}} .
\end{aligned}
$$


We now substitute $(4.28)^{*}$ and $(4.29)^{*}$ in $(4.19)^{*}$ to obtain

$$
\begin{aligned}
J(x)= & \frac{(-1)^{h}}{2 N}\left(\frac{2 \pi}{x}\right)^{\frac{2 h+1}{2 N}} \sum_{k=0}^{r-1} \frac{(-1)^{r-k-1} B_{r-k-1}^{(r)}(\alpha)}{k !(r-k-1) !}(2 \pi)^{-k} \\
& \times \sum_{l=0}^{k}(-1)^{k-l} Q_{l, k}\left(\frac{1}{2 N}, \frac{2 N-2 h-1}{2 N}\right)(2 \pi)^{l}\left(\frac{2 \pi}{x}\right)^{\frac{l}{2 N}} \\
& \times \sum_{j=-(N-1)}^{N-1} \sum_{a=1}^{l+1}(a-1) ! S(l+1, a) \sum_{n=1}^{\infty} n^{\frac{2 h+1+l}{2 N}-k-1} \\
& \times(Z(n, a, j, l, k)+\overline{Z(n, a, j, l, k)})
\end{aligned}
$$

where

$$
Z(n, a, j, l, k)=\frac{\exp \left(-i B^{*}(n, j, l, k)-a A(n / x) \xi_{j}\right)}{\left(2 \sinh \left(A(n / x) \xi_{j}\right)\right)^{a}} .
$$

Hence

$$
Z(n, a, j, l, k)+\overline{Z(n, a, j, l, k)}=B / A,
$$

say, where

$$
A=\left(\cosh \left(2 A\left(\frac{n}{x}\right) a_{j}\right)-\cos \left(2 A\left(\frac{n}{x}\right) b_{j}\right)\right)^{a},
$$

and $B$ is the sum of

$$
2^{-a} e^{-i B^{*}(n, j, l, k)}\left(e^{-2 i A(n / x) b_{j}}-e^{-2 A(n / x) a_{j}}\right)^{a}
$$

and its conjugate, or twice the real part of (4.33), namely

$$
B=2^{1-a} \Re\left\{e^{-i B^{*}(n, j, l, k)}\left(e^{-2 i A(n / x) b_{j}}-e^{-2 A(n / x) a_{j}}\right)^{a}\right\} .
$$

Now, combining (4.33) and (4.35) in (4.32) gives $f^{*}(n, a, j, l, k, x)$, defined in $(4.2)^{*}$, and the proof is complete.

Proof of Lemma 4.1. We expand $[a x+b]_{k}$ by (3.2) and the binomial theorem to obtain

$$
[a x+b]_{k}=\sum_{\nu=0}^{k} s(k, \nu)(a x+b)^{\nu}=\sum_{\nu=0}^{k} s(k, \nu) \sum_{j=0}^{\nu}\left(\begin{array}{l}
\nu \\
j
\end{array}\right) a^{j} b^{\nu-j} x^{j} .
$$

Then expand $x^{j}$ using (4.8) to obtain

$$
[a x+b]_{k}=\sum_{\nu=0}^{k} s(k, \nu) \sum_{j=0}^{\nu}\left(\begin{array}{l}
\nu \\
j
\end{array}\right) a^{j} b^{\nu-j} \sum_{l=0}^{j} S(j, l)[x]_{l} .
$$


Changing the order of summation, we deduce that

$$
[a x+b]_{k}=\sum_{l=0}^{k}\left(\sum_{j=l}^{k} S(j, l) a^{j} \sum_{\nu=j}^{k}\left(\begin{array}{l}
\nu \\
j
\end{array}\right) s(k, \nu) b^{\nu-j}\right)[x]_{l} .
$$

Using the identity $[6$, p. 214]

$$
s(k, \nu)=s(k+1, \nu+1)+k s(k, \nu+1)
$$

and the definition $(3.17)$ of $c_{r, l}(b)$, we conclude that the coefficient of $[x]_{l}$ in (4.36) coincides with $Q_{l, k}(a, b)$ as given at the beginning of this section, whence the assertion follows from the identity $(x)_{k}=(-1)^{k}[-x]_{k}$.

Remark 4. (i) Presumably it is the integral (4.17) that prevented Katsurada from investigating further values of the multiple Hurwitz zeta-function. Indeed the integrand is of the form $(0 \leq a \leq 2 N-1)$

$$
\frac{\Gamma\left(\frac{s}{2 N}+\frac{a}{2 N}+k\right)}{\Gamma\left(\frac{s}{2 N}+\frac{a}{2 N}\right)} \Gamma(s) Y^{-s} .
$$

For general $N$, we have by the multiplication formula

$$
\Gamma(s)=\frac{(2 N)^{s-1 / 2}}{(2 \pi)^{N-1 / 2}} \Gamma\left(\frac{s}{2 N}\right) \Gamma\left(\frac{s}{2 N}+\frac{1}{2 N}\right) \ldots \Gamma\left(\frac{s}{2 N}+\frac{2 N-1}{2 N}\right),
$$

one of which cancels the denominator, and we are left with a product of gamma functions. For $N=1, a=1$, the duplication formula yields a nice cancellation and what remains is

$$
\Gamma\left(\frac{s+1}{2}+k\right) \Gamma\left(\frac{s}{2}\right),
$$

whence the Mellin inversion can be treated by the inverse Heaviside integral.

(ii) Putting $f(x)=[a x+b]_{n}$, we claim that the formula in Lemma 4.1 is the Taylor-Newton interpolation formula [6, p. 221]

$$
f(x)=\sum_{k=0}^{n} \frac{\Delta^{k} f(0)}{k !}[x]_{k}
$$

where $\Delta^{k}$ denotes the $k$ th difference operator.

Acknowledgements. The authors would like to thank Professor Masanori Katsurada for sending his paper [14]. They thank the referee for his many important comments which vastly improved the presentation of the paper.

\section{References}

[1] E. W. Barnes, On the theory of multiple gamma function, Trans. Cambridge Philos. Soc. 19 (1904), 374-425. 
[2] B. C. Berndt, Ramanujan's Notebooks. Part II, Springer, New York, 1989 (esp. p. 276).

[3] B. C. Berndt and R. Evans, Chapter 15 of the Ramanujan Second Notebook: Part II, Modular forms, Acta Arith. 47 (1986), 123-142.

[4] J.-S. Choi, On a generalization of the Hurwitz zeta function $\zeta(s, a)$, Indian J. Pure Appl. Math. 23 (1992), 83-91.

[5] —, Explicit formulas for Bernoulli polynomials of order n, ibid. 27 (1996), 667-674.

[6] L. Comtet, Advanced Combinatorics, Reidel, Dordrecht, 1974.

[7] S. Egami, A $\chi$-analogue of a formula of Ramanujan for $\zeta(1 / 2)$, Acta Arith. 69 (1995), 189-191.

[8] A. Erdélyi (ed.), Higher Transcendental Functions, Vol. II, McGraw-Hill, New York, 1953.

[9] S. Kanemitsu, M. Katsurada and M. Yoshimoto, On the Hurwitz-Lerch zeta function, Aequationes Math. 59 (2000), 1-19.

[10] S. Kanemitsu, H. Kumagai and M. Yoshimoto, Sums involving the Hurwitz zeta function, Ramanujan J. 5 (2001), 5-19.

[11] S. Kanemitsu, Y. Tanigawa and M. Yoshimoto, On rapidly convergent series for the Riemann zeta-values via the modular relation, preprint.

[12] - , - - On zeta- and L-function values at special rational arguments via the modular relation, in: Proc. Internat. Conference on SSFA, Vol. 1, 2001, 21-42.

[13] - - - - On Dirichlet $L$-function values at rational arguments, preprint.

[14] M. Katsurada, On an asymptotic formula of Ramanujan for a certain theta-type series, Acta Arith. 97 (2001), 157-172.

[15] D. Klusch, On Entry 8 of Chapter 15 of Ramanujan's Notebook II, ibid. 58 (1991), 59-64.

[16] N. S. Koshlyakov, Investigation of some questions of analytic theory of the rational and quadratic fields, I-III, Izv. Akad. Nauk SSSR, Ser. Mat. 18 (1954), 113-144, 213-260, 307-326; Errata 19 (1955), 271 (in Russian).

[17] Hj. Mellin, Die Dirichletschen Reihen die zahlentheoretischen Funktionen und unendlichen Produkte von endlichen Geschlecht, Acta Soc. Sci. Fenn. 31 (1902), 1-48.

[18] J. Riordan, Combinatorial Identities, Krieger, New York, 1979.

[19] I. Vardi, Determinants of Laplacians and multiple gamma functions, SIAM J. Math. Anal. 19 (1988), 493-507.

[20] E. T. Whittaker and G. N. Watson, A Course of Modern Analysis, Cambridge Univ. Press, London, 1927.

Graduate School of Advanced Technology

University of Kinki, Iizuka

Fukuoka, 820-8555, Japan

E-mail: kanemitu@fuk.kindai.ac.jp
Graduate School of Mathematics

Nagoya University

Nagoya, 464-8602, Japan

E-mail: tanigawa@math.nagoya-u.ac.jp x02001n@math.nagoya-u.ac.jp

Received on 12.7.2001

and in revised form on 26.4.2002 